\title{
An application in identifying high-risk populations in alternative tobacco product use utilizing logistic regression and CART: a heuristic comparison
}

\author{
Yang Lei ${ }^{1 *}$, Nikki Nollen², Jasjit S Ahluwahlia ${ }^{3}$, Qing Yu ${ }^{1}$ and Matthew S Mayo ${ }^{1}$
}

\begin{abstract}
Background: Other forms of tobacco use are increasing in prevalence, yet most tobacco control efforts are aimed at cigarettes. In light of this, it is important to identify individuals who are using both cigarettes and alternative tobacco products (ATPs). Most previous studies have used regression models. We conducted a traditional logistic regression model and a classification and regression tree (CART) model to illustrate and discuss the added advantages of using CART in the setting of identifying high-risk subgroups of ATP users among cigarettes smokers.

Methods: The data were collected from an online cross-sectional survey administered by Survey Sampling International between July 5, 2012 and August 15, 2012. Eligible participants self-identified as current smokers, African American, White, or Latino (of any race), were English-speaking, and were at least 25 years old. The study sample included 2,376 participants and was divided into independent training and validation samples for a hold out validation. Logistic regression and CART models were used to examine the important predictors of cigarettes + ATP users.
\end{abstract}

Results: The logistic regression model identified nine important factors: gender, age, race, nicotine dependence, buying cigarettes or borrowing, whether the price of cigarettes influences the brand purchased, whether the participants set limits on cigarettes per day, alcohol use scores, and discrimination frequencies. The C-index of the logistic regression model was 0.74 , indicating good discriminatory capability. The model performed well in the validation cohort also with good discrimination (c-index $=0.73$ ) and excellent calibration (R-square $=0.96$ in the calibration regression). The parsimonious CART model identified gender, age, alcohol use score, race, and discrimination frequencies to be the most important factors. It also revealed interesting partial interactions. The $c$-index is 0.70 for the training sample and 0.69 for the validation sample. The misclassification rate was 0.342 for the training sample and 0.346 for the validation sample. The CART model was easier to interpret and discovered target populations that possess clinical significance.

Conclusion: This study suggests that the non-parametric CART model is parsimonious, potentially easier to interpret, and provides additional information in identifying the subgroups at high risk of ATP use among cigarette smokers.

Keywords: Survey sampling, Stratified samples, Logistic regression, CART, Partial interaction

\section{Background}

Recent years have witnessed increased tobacco control policies at both the state and national level [1-3]. Most of these efforts are aimed at cigarette smoking [1]. The net effects of these policies include decreased cigarettes consumption, as well as a shift in the type of tobacco products used $[4,5]$. The use of alternative forms of

\footnotetext{
*Correspondence: ylei@kumc.edu

'Department of Biostatistics, The University of Kansas Medical Center, Kansas City, KS, USA

Full list of author information is available at the end of the article
}

tobacco products (ATPs), such as large cigars, little cigars, cigarillos, pipes, hand-rolled cigarettes, smokeless tobacco, and hookahs, are increasing in prevalence [6,7]. About $8 \%-38 \%$ of U.S. daily smokers and as many as $44 \%$ of non-daily smokers (smoke on some but not all days) are ATP users [5-10], defined as anyone who uses cigarettes and alternative forms of tobacco. These tobacco products have been promoted as less addictive and less harmful than cigarettes $[11,12]$. Nevertheless, data suggest that use of these products could be associated with higher nicotine dependence and may contribute to increased

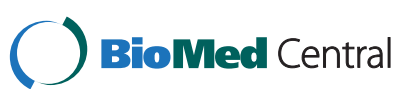


risks for diseases caused by tobacco, such as cancer and heart disease [13].

It is of utmost importance to identify individuals who are at high risk of using both cigarettes and ATPs. Research subjects in previous studies have been predominately White $[8,9,14,15]$ and most existing studies have used traditional regression approaches to identify important factors associated with ATP use. Although regression methods can test a priori specified interaction effects, it lacks the ability to capture unspecified, complex inter-relationships across factors. Classification and Regression Trees model (CART) can address these limitations by revealing unspecified inter-relationships through an easily interpretable tree diagram. Few studies have applied CART modeling to tobacco research $[16,17]$. In this paper we used data from a cross-sectional survey of smokers and conducted the most commonly used logistic regression method and relatively underused CART method, and described the strength and limitations of these two statistical approaches in identifying cigarette smokers at highest-risk for ATP use.

\section{Methods Study population}

The data was collected through a cross-sectional survey administered through an online panel survey service, Survey Sampling International (SSI), between July 5, 2012 and August 15, 2012. Ethical approval was granted by the University of Minnesota Institutional Review Board. Participants were presented with a written informed consent page prior to completing the screener. Only participants who indicated their consent were directed to the study questions. Eligible participants self-identified as current smokers, African American, White, or Latino (of any race), were English-speaking, and were at least 25 years old. The study sample contained 2,376 participants balanced by the three racial/ethnic groups across smoking frequencies (daily and nondaily smoking): 794 African Americans, 786 Latinos, and 796 whites. Among them, 1,220 participants (51.35\%) were cigarettes + ATP users who used both cigarettes and other tobacco products and $1,156(48.65 \%)$ were cigarettes-only users. Variable domains in this study included: demographics, tobacco characteristics, cost concerns, harm reduction efforts, and psychosocial variables. There was minimal missing data, about $4.3 \%$ subjects were missing one variable (income), and therefore imputation was not necessary. Chi-square tests were used to test the unadjusted effects of categorical variables and T-tests were used to test continuous variables (Table 1$)$.

\section{Training and validation data sets}

The large sample size allowed for the use of a hold-out validation to obtain independent training and validation data sets [18-23]. The data was partitioned by random sampling, stratifying by cigarettes + ATP use and race/ ethnicity to ensure the balance we designed. Training sample contained 1,584 participants (two thirds of the sample) and was used to derive the model. The remaining data contained 792 participants (one third of the sample) and were used to evaluate the predictive ability of the final model. The training and validation samples were compared to ensure the differences between the two were negligible (Table 2).

\section{Analysis \\ Logistic regression}

Logistic regression is a traditional way to identify important factors for binary outcomes. The Akaike Information Criterion (AIC) is widely recommended as a model selection criterion [18]. To avoid the technical difficulty of comparing AICs from all possible variable combinations, we followed a model selection strategy recommended by Frank E. Harrell to trim the potential models [18] and then picked the minimal AIC model from the potential models as the final model. The selection process started with all potential factors. Predicted values from the logistic regression were then regressed on all covariates, with the model explaining $100 \%$ of the variance. Backward selection based on $R^{2}$ was used to select a parsimonious set of variables. The contribution of each covariate in the multivariable model was ranked, and variables with the smallest contribution to the model were sequentially eliminated. This iterative process continued until further variable elimination led to a greater than 5\% loss in model prediction, as compared with the initial model. The remaining covariates comprised the parsimonious model and explained over $95 \%$ of the variance of the full model. Finally, we compared AIC values of neighborhood models around the model we obtained in the last step and the minimum AIC model was selected as the final model. This selection strategy supports inclusion of only variables that provide incremental prognostic value, avoids over-fitting, and maximizes the potential usefulness of the model. Besides this model selection strategy, we examined backward selections based on p-value with 0.15 as the threshold to enter and 0.05 as the threshold to stay in the model. Both approaches identified the same model.

Predicted values using the model estimates from the training cohort were generated for the validation cohort and the c-index was then calculated based on the proportion of concordance. The predicted values were ranked and cut into deciles. The calibration plot was graphed comparing the average predicted probabilities with the observed average probabilities. A calibration regression on observed mean probabilities was performed using predicted mean probabilities to check the strength of correlation between the predicted and the observed average probabilities across deciles. 
Table 1 Univariate differences between smokers who use cigarettes in combination with alternative tobacco product (cigarettes + ATP) compared to those who use cigarettes only

\begin{tabular}{|c|c|c|c|}
\hline & $\begin{array}{l}\text { Cigarettes + ATP } \\
(n=1,220)\end{array}$ & $\begin{array}{l}\text { Cigarettes only } \\
(n=1,156)\end{array}$ & $\mathrm{p}$ value \\
\hline \multicolumn{4}{|l|}{ Demographics } \\
\hline Male & $662(27.9 \%)$ & $332(14.0 \%)$ & $<0.001$ \\
\hline Age $( \pm S D)$ & $40.24 \pm 11.64$ & $45.85 \pm 12.62$ & $<0.001$ \\
\hline Race & & & $<0.001$ \\
\hline African American & $436(18.4 \%)$ & $358(15.1 \%)$ & \\
\hline Latino & $455(19.1 \%)$ & $331(13.9 \%)$ & \\
\hline White & $329(13.8 \%)$ & $467(19.7 \%)$ & \\
\hline Education, \% college graduate or higher & $474(19.9 \%)$ & $364(15.3 \%)$ & $<0.001$ \\
\hline Income, $\%<\$ 1800 /$ month & $480(20.2 \%)$ & $463(19.5 \%)$ & 0.725 \\
\hline \multicolumn{4}{|l|}{ Tobacco Characteristics } \\
\hline Smoking status (\%) & & & $<0.001$ \\
\hline Nondaily & $673(28.3 \%)$ & $528(22.2 \%)$ & \\
\hline Daily light (1-10 cpd) & $259(10.9 \%)$ & $319(13.4 \%)$ & \\
\hline Daily heavy $(11+\mathrm{cpd})$ & $288(12.1 \%)$ & $309(13.0 \%)$ & \\
\hline Menthol smoker & 737 (31.0\%) & $623(26.2 \%)$ & 0.001 \\
\hline Cigarettes per day, mean $( \pm \mathrm{SD})$ & $9.30 \pm 8.70$ & $10.14 \pm 8.52$ & 0.017 \\
\hline Time to first cigarette, $\%$ within 30 minutes of waking & $720(30.3 \%)$ & $629(26.5 \%)$ & 0.024 \\
\hline 24 hour quit attempts in last 12 months, mean $( \pm S D)$ & $5.50 \pm 9.53$ & $5.94 \pm 11.79$ & 0.451 \\
\hline \multicolumn{4}{|l|}{ Cost } \\
\hline Price of cigs influenced them to smoke less, $\%$ yes & $726(30.6 \%)$ & $644(27.1 \%)$ & 0.061 \\
\hline Price of cigs influenced where they buy cigs, $\%$ yes & $840(35.4 \%)$ & $826(34.8 \%)$ & 0.166 \\
\hline Price of cigs influenced the brand they buy, $\%$ yes & $590(24.8 \%)$ & $455(19.1 \%)$ & $<0.001$ \\
\hline Buy versus borrow cigs, $\%$ buy all cigs they smoke & $683(28.7 \%)$ & $824(34.7 \%)$ & $<0.001$ \\
\hline \multicolumn{4}{|l|}{ Harm Reduction } \\
\hline Trying to cut down on cigs smoke, $\%$ yes & $862(36.3 \%)$ & $818(34.4 \%)$ & 0.955 \\
\hline Limit cpd to decrease health risk, \% yes & $596(25.1 \%)$ & $505(21.3 \%)$ & 0.012 \\
\hline Limit smoking in last year to decrease health risks, \% always or often & $360(15.2 \%)$ & $356(15.0 \%)$ & 0.494 \\
\hline \multicolumn{4}{|l|}{ Psychosocial } \\
\hline Depression score, mean $( \pm \mathrm{SD})^{\mathrm{a}}$ & $2.14 \pm 1.83$ & $1.80 \pm 1.84$ & $<0.001$ \\
\hline Alcohol score, mean $( \pm S D)^{b}$ & $4.64 \pm 3.10$ & $3.30 \pm 2.98$ & $<0.001$ \\
\hline Discrimination score, mean $( \pm S D)^{c}$ & $8.28 \pm 6.72$ & $5.85 \pm 5.66$ & $<0.001$ \\
\hline
\end{tabular}

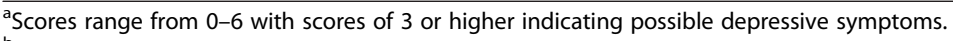

${ }^{b}$ Scores range from $0-12$ with scores of $\geq 4$ for men and $\geq 3$ for women indicating possible alcohol misuse.

'Scores range from 0-25 with higher scores indicating greater frequency of discrimination in daily life.

\section{Classification and Regression Tree (CART) model}

Although the logistic regression model provides knowledge of important profile characteristics, it lacks the ability to identify unknown, and therefore, unspecified interaction effects. The interpretation of parameter estimates is based on the fact of controlling for all other covariates. To address these problems, we built Classification and Regression Tree models (CART) in SAS Enterprise Miner version $12.3[24,25]$. CART is a nonparametric method that identifies mutually exclusive and exhaustive subgroups. Members within each subgroup share the same characteristics that influence the probability of belonging to the interested response group [26]. CART produces a model structure that resembles an upsidedown tree. The tree starts with the parent node, and the parent node contains the entire population. The CART algorithm examines all possible independent variables according to a predetermined splitting rule and divides the parent node into two child nodes; the child nodes can be further divided into more child nodes. There 
Table 2 Univariate Differences between training sample and validation sample

\begin{tabular}{|c|c|c|c|}
\hline & $\begin{array}{l}\text { Training } \\
(n=1584)\end{array}$ & $\begin{array}{l}\text { Validation } \\
(\mathrm{n}=792)\end{array}$ & $P$ value \\
\hline \multicolumn{4}{|l|}{ Demographics } \\
\hline Male & $657(27.7 \%)$ & $337(14.2 \%)$ & 0.617 \\
\hline Age $( \pm S D)$ & $42.94 \pm 12.39$ & $43.03 \pm 12.5$ & 0.880 \\
\hline Race & & & 0.997 \\
\hline African American & $530(22.3 \%)$ & $264(11.1 \%)$ & \\
\hline Latino & $524(22.1 \%)$ & $262(11.0 \%)$ & \\
\hline White & $530(22.3 \%)$ & $266(11.2 \%)$ & \\
\hline Education, \% college graduate or higher & $550(23.1 \%)$ & $288(12.1 \%)$ & 0.430 \\
\hline Income, $\%<\$ 1800 /$ month & $614(25.8 \%)$ & $329(13.8 \%)$ & 0.192 \\
\hline \multicolumn{4}{|l|}{ Tobacco Characteristics } \\
\hline Smoking status (\%) & & & 0.263 \\
\hline Nondaily & $799(33.6 \%)$ & $402(16.9 \%)$ & \\
\hline Daily light $(1-10 \mathrm{cpd})$ & $373(15.7 \%)$ & $205(8.6 \%)$ & \\
\hline Daily heavy $(11+\mathrm{cpd})$ & $412(17.3)$ & $185(7.8 \%)$ & \\
\hline Menthol smoker & $899(37.8 \%)$ & $461(19.4 \%)$ & 0.500 \\
\hline Cigarettes per day, mean $( \pm S D)$ & $10.03 \pm 9.03$ & $9.06 \pm 7.69$ & 0.009 \\
\hline Time to first cigarette, $\%$ within 30 minutes of waking & $900(37.9 \%)$ & $449(18.9 \%)$ & 0.953 \\
\hline 24 hour quit attempts in last 12 months, mean $( \pm S D)$ & $5.54 \pm 9.87$ & $6.00 \pm 11.93$ & 0.454 \\
\hline \multicolumn{4}{|l|}{ Cost } \\
\hline Price of cigs influenced them to smoke less, $\%$ yes & $920(38.7 \%)$ & $450(18.9 \%)$ & 0.557 \\
\hline Price of cigs influenced where they buy cigs, $\%$ yes & $1100(46.3 \%)$ & $566(23.8 \%)$ & 0.311 \\
\hline Price of cigs influenced the brand they buy, $\%$ yes & $685(28.8 \%)$ & $360(15.2 \%)$ & 0.306 \\
\hline Buy versus borrow cigs, $\%$ buy all cigs they smoke & $1004(42.3 \%)$ & $503(21.2 \%)$ & 0.952 \\
\hline \multicolumn{4}{|l|}{ Harm Reduction } \\
\hline Trying to cut down on cigs smoke, $\%$ yes & $1119(47.1 \%)$ & $561(23.6 \%)$ & 0.924 \\
\hline Limit cpd to decrease health risk, \% yes & $730(30.7 \%)$ & $371(15.6 \%)$ & 0.727 \\
\hline Limit smoking in last year to decrease health risks, \% always or often & $476(20.0 \%)$ & $240(10.1 \%)$ & 0.899 \\
\hline \multicolumn{4}{|l|}{ Psychosocial } \\
\hline Depression score, mean $( \pm \mathrm{SD})^{\mathrm{a}}$ & $1.99 \pm 1.86$ & $1.96 \pm 1.82$ & 0.683 \\
\hline Alcohol score, mean $( \pm S D)^{b}$ & $4.02 \pm 3.16$ & $3.93 \pm 3.03$ & 0.494 \\
\hline Discrimination score, mean $( \pm S D)^{c}$ & $7.03 \pm 6.30$ & $7.23 \pm 6.44$ & 0.460 \\
\hline
\end{tabular}

${ }^{\text {a }}$ Scores range from $0-6$ with scores of 3 or higher indicating possible depressive symptoms.

${ }^{b}$ Scores range from $0-12$ with scores of $\geq 4$ for men and $\geq 3$ for women indicating possible alcohol misuse.

cScores range from $0-25$ with higher scores indicating greater frequency of discrimination in daily life.

are many splitting rules, and they all begin with defining the impurity of a node [26]. The impurity function measures the extent of difference/similarity for a node containing data points from possible different classes. A node that has no impurity would have no variability (e.g. all cigarettes-only smokers, or all cigarettes + ATP smokers). The highest impurity is achieved when $\mathrm{p}(\mathrm{k} \mid \mathrm{t})=0.5$, where $\mathrm{p}(\mathrm{k} \mid \mathrm{t})$ is defined as the conditional probability of belonging to class $\mathrm{k}$ given in node $\mathrm{t}$. Although the impurity functions may vary, all splitting rules select the split that has the largest difference between the impurity of the parent node and a weighted average of the impurity of the two child nodes. The Gini splitting rule was recommended most for binary outcomes [25]. This rule maximizes the following improvement of impurity function:

$$
x_{j} \leq x_{j}^{R}, j=1, \ldots, M\left[-\sum_{k=1}^{\arg \max } p^{2}\left(k \mid t_{p}\right)+P_{l} \sum_{k=1}^{K} p^{2}\left(k \mid t_{l}\right)+P_{r} \sum_{k=1}^{K} p_{2}\left(k \mid t_{r}\right)\right]
$$

$p(k \mid t)$ : conditional probability of dependent variable $=k$ given node $t$ subscript p: parent node 
subscript $r$ : child right node

subscript l: child left node

$P_{l}$ : probability in the left child node

$P_{r}$ : probability in the right child node (note: $P_{l}+\mathrm{P}_{r}=1$ )

$x_{j}^{R}$ : best splitting value of variable $x_{j}$

M: number of potential independent variables

K: level of dependent variables. For binary outcomes, $\mathrm{K}=2$

The larger the value of the improvement in impurity function, the greater difference between the two child nodes with respect to the prevalence of the dependent measure. The CART procedure selects the independent variable and the splitting cutoff of the continuous independent variable to maximize the improvement at each step. The tree grows as child nodes are divided into more child nodes. The terminal nodes are where predictions and inferences are made.

It is clear that different samples would produce different trees. One common way to assess how different the trees could be is using training and validation samples. To facilitate comparisons, the same set of training and validation samples were used in logistic regression model and CART model. In CART model, misclassification rates from both the training sample and the validation sample were compared to ensure the model is stable.

The maximum tree with the minimum misclassification error was examined and the misclassification error graph showed that it contained insignificant nodes, which reduced the misclassification error marginally but increased the complexity greatly. A popular stopping strategy was applied by predefining the minimum number of points in the terminal node to control the size of the tree [26]. The minimum node size was set to be $10 \%$ of the training sample size or about 150 subjects in our study. Models were assessed to identify a parsimonious tree that produces non-trivial results with acceptable misclassification rates.

\section{Results}

Logistic regression model

The final model consisted of nine variables (Table 3). Males had the strongest association with being a cigarettes + ATP user vs. cigarettes-only user (adjusted OR 2.66, 95\% CI 2.12 - 3.33). African Americans and Latino were more likely to be cigarettes + ATP users compared to whites (adjusted OR 1.58, 95\% CI $1.21-2.07$ and adjusted OR 1.52 , 95\% CI 1.16 - 1.99, respectively). Individuals with higher nicotine dependence were more likely to be cigarettes + ATP users (adjusted OR 1.51, 95\% CI 1.20 - 1.90). Participants who buy their cigarettes were less likely to be cigarettes + ATP users compared to those who borrow cigarettes from others (adjusted OR 0.617, 95\% CI 0.49 0.78 ). Individuals who were more sensitive to the price of cigarettes were more likely to be cigarettes + ATP users (adjusted OR 1.43, 95\% CI 1.14 -1.79). Individuals who set limit on cigarettes per day were more likely to be cigarettes + ATP users (adjusted OR 1.30, 95\% CI 1.04 - 1.62). Individuals with higher alcohol scores were more likely to be cigarettes + ATP users (adjusted OR 1.10, 95\% CI 1.064-1.145). Older people were less likely to use cigarettes + ATPs (adjusted OR 0.97, 95\% CI 0.96-0.98). Higher discrimination scores were associated with higher probability of using cigarettes + ATPs (adjusted OR 1.03, 95\% CI 1.01 - 1.05). The C-index of the final model was 0.74 , indicating good discriminatory capacity (Figure 1).

\section{Model validation}

Participants were similar in terms of all profile characteristics (Table 2), except that participants in the validation cohort smoked about 1 cigarette per day less than the training cohort (10 vs. $9, \mathrm{p}=0.009)$. The model performed well in the validation cohort with good discrimination (c-index $=0.73$ ) and excellent calibration with an intercept of 0.018 ( $\mathrm{p}$-value for difference

Table 3 Results from logistic regression on the training cohort: parameter estimates and odds ratios

\begin{tabular}{lllll}
\hline Parameter & Estimate & Odds ratio & 95\% CL for OR & P-value \\
\hline Intercept & -0.2617 & NA & NA & 0.3497 \\
Age & -0.0265 & 0.974 & $(0.964,0.983)$ & $<.0001$ \\
Male & 0.9766 & 2.655 & $(2.118,3.329)$ & $<.0001$ \\
Buy vs. Borrow & -0.4832 & 0.617 & $(0.486,0.783)$ & $<.0001$ \\
Alcohol & 0.0986 & 1.104 & $(1.064,1.145)$ & $(1.144,1.788)$ \\
Price influenced the brand they buy & 0.3579 & 1.430 & $(1.208,2.066)$ & 0.0001 \\
African American vs. white & 0.4576 & 1.580 & $(1.155,1.994)$ & 0.0008 \\
Latino vs. white & 0.4170 & 1.517 & $(1.007,1.045)$ & 0.0028 \\
Discrimination & 0.0259 & 1.026 & $(1.197,1.897)$ & 0.0065 \\
Time to first cig less than 30 min & 0.4100 & 1.507 & $(1.041,1.619)$ & 0.0203 \\
\hline Limit cigarettes per day & 0.2612 & 1.299 &
\end{tabular}




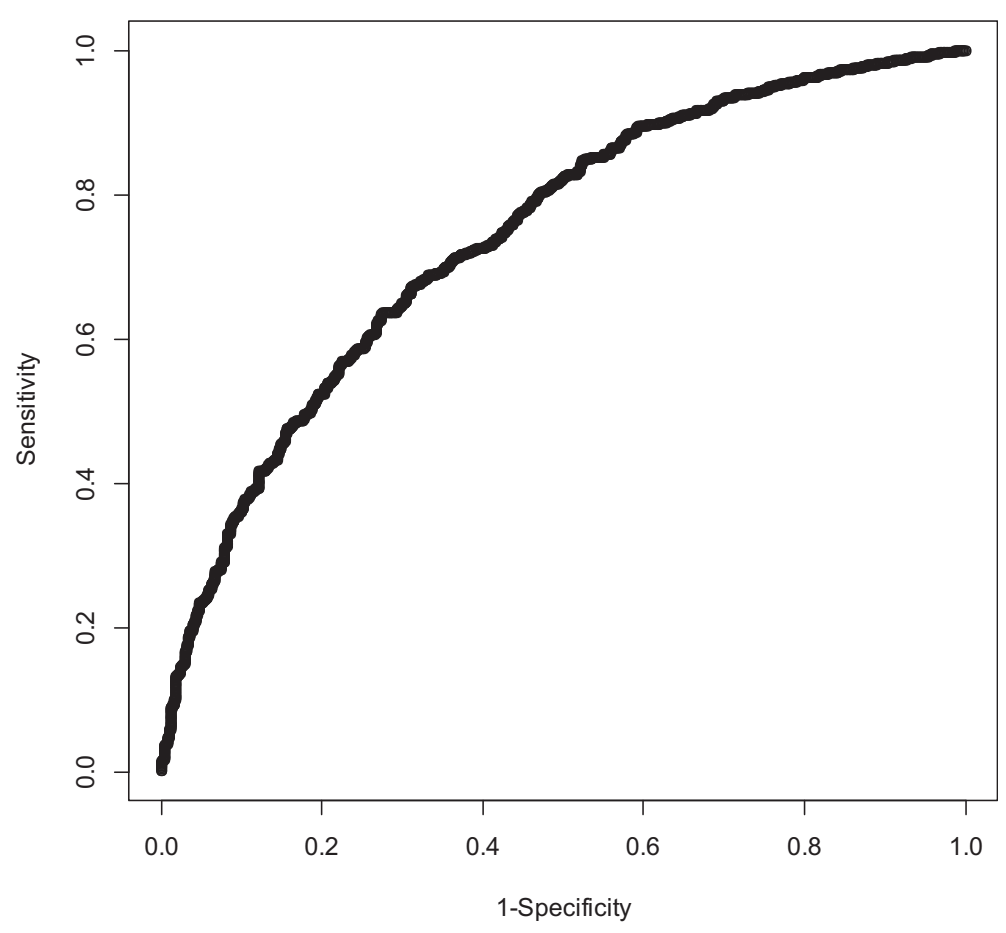

Figure 1 ROC curve from logistic regression on the training sample. Area under the curve $=0.7403$.

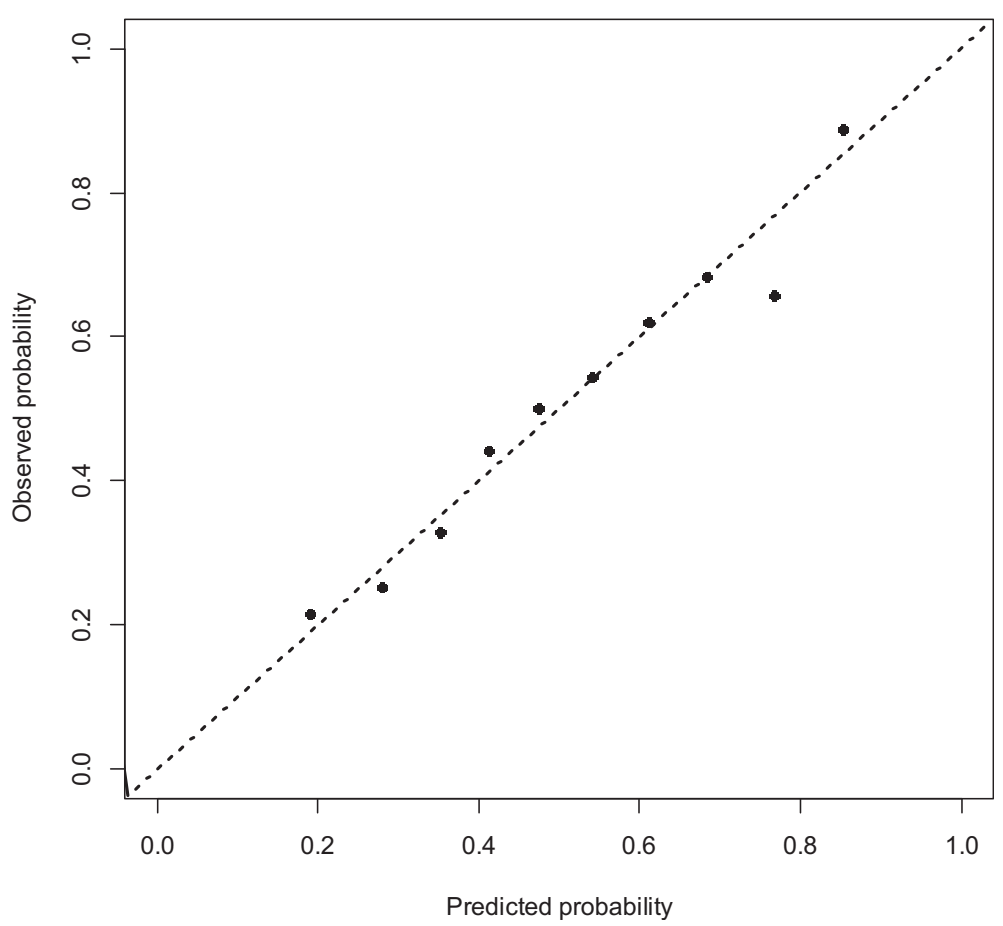

Figure 2 Calibration plot from the validation sample. Observed vs. predicted probabilities across deciles, $R^{2}=0.96$ 
from $0=0.65$ ) and a slope $=0.96$ ( $\mathrm{p}$-value for difference from $1=0.58$ ). The R-square for the calibration regression was 0.96 and the Pearson correlation coefficient was 0.98 (p-value $<0.0001$ ) (Figure 2).

\section{Classification and Regression Tree (CART) model}

Figure 3 shows the final tree results using the stopping rule of minimum node size no less than 150 . The same independent training and validation samples were used as in the logistic regression. The misclassification rate was 0.342 for the training sample and 0.346 for the validation sample. The $\mathrm{C}$-index was 0.70 for the training sample and 0.69 for the validation sample.

Males were more likely to be cigarettes + ATP users, especially when they were moderate to heavy drinkers (alcohol use score $>2$ ). A male with a 3 or higher alcohol score had $73.5 \%$ probability of being a cigarettes + ATP user. Females were less likely to be cigarettes + ATP users, especially when they were older. Female participants aged 46 or older had a $29.0 \%$ probability of being cigarettes + ATP users. Among females age 45 years or younger, Latino and African Americans were more likely to be cigarettes + ATP users compared to whites. $37.2 \%$ of White females aged 45 years or younger were cigarettes + ATP users. Latino and African American females aged 45 or younger, who also experienced greater discrimination were more likely to be cigarettes + ATP users, about $62.2 \%$ probability if their discrimination score was greater than 6 (Figure 3). Interestingly, age, race, and discrimination effects that impacted female participants did not play important roles for males. Alcohol scores increased the risk of cigarettes + ATP use for males but were not important for females. These indicated informative interaction patterns to examine the profile characteristics of cigarettes + ATP users.

\section{Limitations}

A hold-out validation strategy was used in this study to obtain independent training and validation datasets. The reduced data can result in an enlarged variance. Although this method is reasonable in this study because the sample size is large, other validation strategies, such as $\mathrm{k}$-fold cross validation, which uses overlapped training data, may achieve more accurate performance estimation. We used a method suggested by Harrell [18] to trim potential models and then compared AIC of these potential

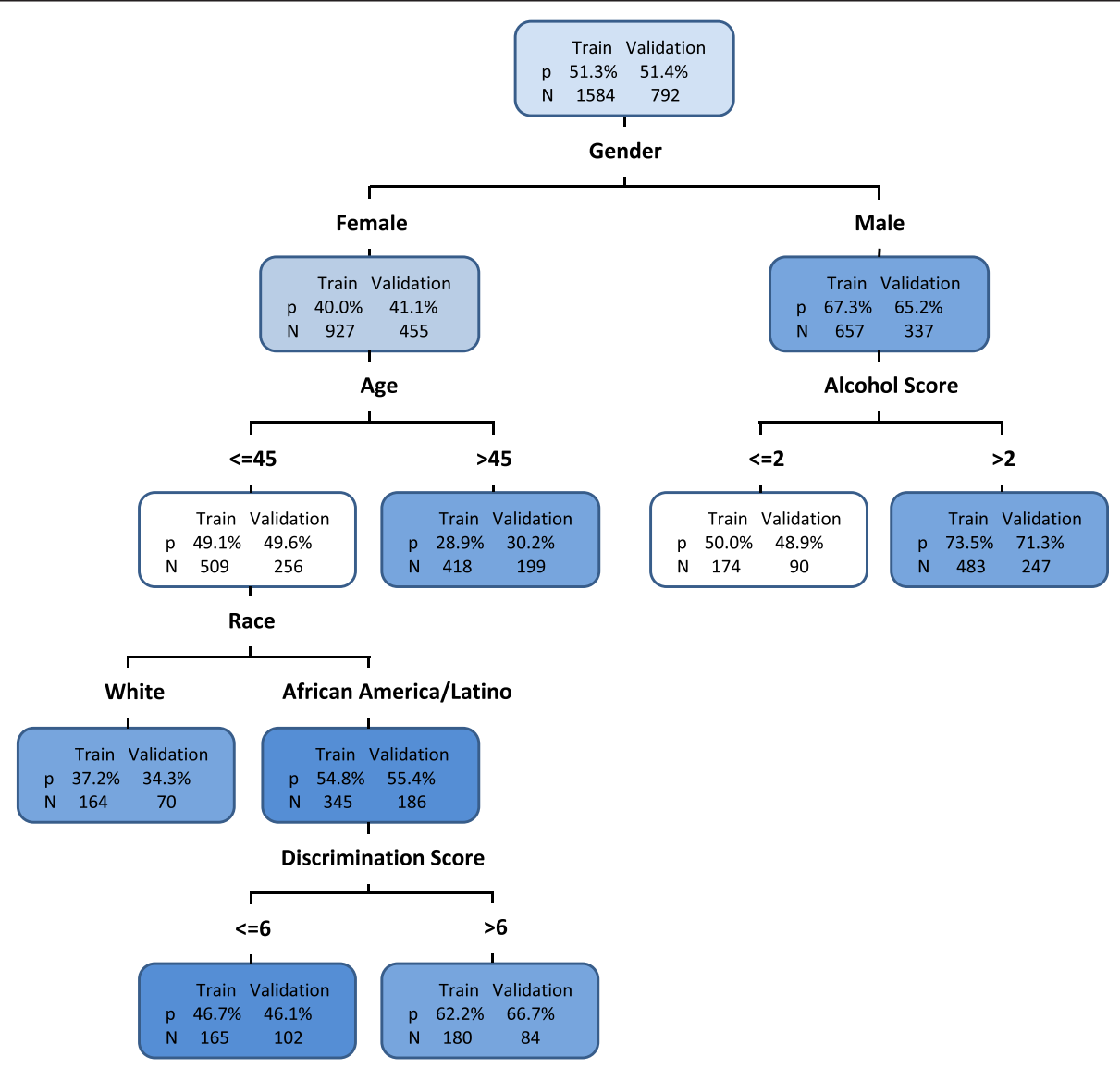

Figure 3 Classification and Regression Tree model for predicting Cig + ATP users. The number of participants (N) and the probability of Cig + ATP users (P) are given inside of each node for both training and validation samples. 
models to obtain the final model. Other model selection strategies, such as LASSO and ridge regression were not compared with this method.

\section{Discussion}

The CART model identified the five most important factors: gender, alcohol scores, age, race, and discrimination scores. The logistic regression model identified nine variables: the same five as the CART model, and additionally, whether the participant buys or borrows cigarettes from others, whether the participant limits cigarettes per day, price influences, and nicotine dependence. Therefore, the logistic regression model expanded the variable pool from the CART model.

The logistic regression model results in higher C-index than the CART model $(0.74$ versus 0.70 for the training sample and 0.73 versus 0.69 for the validation sample). However, the C-index from the CART model was not directly comparable to that in the logistic regression model because the classifiers varied across different subgroups in the CART model due to partial interaction effects. On the other hand, logistic regression models lack the ability to identify unspecified, complex interrelationships between factors. In studies where interaction effects are unclear, it is impractical to test all potential interaction effects in logistic regression models. However, there might be potential inter-relationships, especially among demographic, psychosocial, and economic factors. Even if the logistic regression model achieves good model fit, we could still miss interaction effects that are significant to clinical practice. CART analysis is efficient to address these problems and it is easy to perform with available statistical software. It has great flexibility of building a model that can be easily interpreted through pictorial illustration, without pulling in too much complexity. CART can be considered as complementary to logistic regression models and the result from CART revealed clearly classified high-risk populations of ATP use among cigarette smokers.

\section{Conclusions}

The growing trend of ATP use could ultimately cut down the effect of tobacco control efforts that we have seen in recent years. Compared to the traditional logistic regression model, our CART model is more straightforward in classifying individuals at high risk of using cigarettes + ATPs. This model identified fewer factors associated with cigarettes + ATP use and revealed partial interactions that are not easy to find in logistic regression, thus provided clearer direction for identification and treatment in clinical practice. In general, the CART methodology can be used to classify high risk or at need groups for identification for treatment protocols including behavioral interventions.

\section{Abbreviations}

CART: Classification and Regression Trees; ATP: Alternative tobacco product; AIC: Akaike Information Criterion.

\section{Competing interests}

The authors declare that they have no competing interests. Dr. Ahluwalia is funded by Pfizer's Global Research Awards for Nicotine Dependence. Dr. Ahluwalia is also supported in part by the National Institute for Minority Health Disparities (NCMHD/NIH - 1P60MD003422). Pfizer had no role in the design and conduct of the study; in the collection, analysis, and interpretation of the data; or in the preparation, review, or approval of the manuscript.

\section{Authors' contributions}

MM and NN contributed to the study design. YL conducted the study analysis and drafted the manuscript. JSA was the PI of the project, collected the data, and read the draft and contributed to the final manuscript. QY validated the data analysis. All authors revised and approved the final manuscript.

\section{Authors' information}

Dr. Nollen has submitted a clinical paper "Adult Cigarette Smokers at Highest Risk for Alternative Tobacco Product Use Among a Racially/Ethnically and Socioeconomically Diverse Sample" to journal Nicotine and Tobacco Research. That paper used the same dataset and the CART models appeared in this paper also appeared in her paper. However, the clinical paper is for a very different purpose and different audience. Our paper is focused on comparison of two different modeling methods and her paper focused on interpretation of the CART and its relevance to public health research and practice.

\section{Acknowledgements}

We would like to thank Jianghua He for her comments to help improve the study and Andrés Rodriguez for his help to proof read this manuscript.

\section{Author details}

'Department of Biostatistics, The University of Kansas Medical Center, Kansas City, KS, USA. 'Department of Preventive Medicine and Public Health, The University of Kansas Medical Center, Kansas City, KS, USA. ${ }^{3}$ Department of Medicine and Center for Health Equity, The University of Minnesota Medical School, Minneapolis, MN, USA.

Received: 11 August 2014 Accepted: 24 February 2015

Published online: 09 April 2015

\section{References}

1. American Nonsmokers' Rights Foundation. Overview List - How Many Smokefree Laws? http://www.no-smoke.org/pdf/mediaordlist.pdf. [Accessed August 19 2013].

2. H.R. 1256 (111th). Family Smoking Prevention and Tobacco Control Act. Public Law 111-31, 123 Stat. 1776, 2009. http://www.gpo.gov/fdsys/pkg/ PLAW-111publ31/pdf/PLAW-111publ31.pdf. [Accessed Jan 14 2014].

3. Orzechowski W, Walker RC. The Tax Burden on Tobacco. Arlington, VA: Orzechowski and Walker Economic Consulting Firm; 2011.

4. Centers for Disease Control and Prevention. Current cigarette smoking among adults-United States, 2011. MMWR Morb Mortal Wkly Rep. 2012;61:889-94.

5. Kasza KA, Bansal-Travers M, O'Connor RJ, Compton WM, Kettermann A Borek N, et al. Cigarette smokers' use of unconventional tobacco products and associations with quitting activity: findings from the ITC-4 U.S. cohort. Nicotine Tob Res. 2014;16(6):672-81

6. Campbell ML, Bozec LJ, McGrath D, Barrett SP. Alcohol and tobacco co-use in nondaily smokers: an inevitable phenomenon? Drug Alcohol Rev. 2012;31:447-50.

7. McGrath DS, Temporale KL, Bozec LJ, Barrett SP. Polytobacco use in non-daily smokers: an issue requiring greater attention. Prev Med. 2011;53:353-4.

8. Bombard JM, Pederson LL, Nelson DE, Malarcher AM. Are smokers only using cigarettes? exploring current polytobacco use among an adult population. Addict Behav. 2007:32:2411-9.

9. Backinger CL, Fagan P, O'Connell ME, Grana R, Lawrence D, Bishop JA, et al. Use of other tobacco products among U.S. Adult cigarette smokers: prevalence, trends and correlates. Addict Behav. 2008;33:472-89. 
10. Popova L, Ling PM. Alternative tobacco product use and smoking cessation: a national study. Am J Public Health. 2013;103(5):923-30.

11. Jolly DH. Exploring the use of little cigars by students at a historically black university. Prev Chronic Dis. 2008;5:A82.

12. Page JB, Evans S. Cigars, cigarillos, and youth: emergent patterns in subcultural complexes. J Ethn Subst Abus. 2003;2:63-76.

13. Djordjevic MV, Doran KA. Nicotine Content and Delivery across Tobacco Products. Handb Exp Pharmacol. 2009, 61-82.

14. Bombard JM, Pederson LL, Koval JJ, O'Hegarty M. How are lifetime polytobacco users different than current cigarette-only users? results from a Canadian young adult population. Addict Behav. 2009;34:1069-72.

15. Richardson A, Xiao H, Vallone DM. Primary and dual users of cigars and cigarettes: profiles, tobacco use patterns and relevance to policy. Nicotine Tob Res. 2012;14(8):927-32

16. Piper ME, Loh WY, Smith SS, Japuntich SJ, Baker TB. Using decision tree analysis to idnetify risk factors for relapse to smoking. Subst Use Misuse. 2011;46(4):492-510.

17. Swan GE, Javitz HS, Jack LM, Curry SJ, MCAfee T. Heterogeneity in 12-month outcome among female and male smokers. Addiction. 2004;99(2):237-50.

18. Harrell FE. Regression modeling strategies: with application to linear models, logistic regression, and survival analysis. New York: Springer-Verlag; 2001.

19. Larson SC. The shrinkage of the coefficient of multiple correlation. J Educat Psychol. 1931;22:45-55.

20. Mosteller F, Wallace DL. Inference in an authorship problem. J Am Stat Assoc. 1963;1:275-309.

21. Mosteller F, Wallace DL. Data analysis, including statistics. In: Handbook of Social Psychology. Reading, MA: Addison-Wesley; 1968.

22. Refaeilzadeh P, Tang L, Liu H. Cross Validation. http://leitang.net/papers/encycross-validation.pdf. [Accessed Dec 18, 2013.

23. Encyclopedia of statistics. In Quality and Reliability. Ruggeri F, KenettR, Faltin F, ed. Hoboken, New Jersey: Wiley, 2008:315-23.

24. Loh WY. Classification and Regression Trees. Discovery. 2011:14-23

25. Gordon L. Using Classification and Regression Trees (Cart) in Sas Enterprise Miner for Application in Public Health. in SAS Global Forum (2013). http://support.sas.com/resources/papers/proceedings13/089-2013.pdf. [Accessed August 20 2013].

26. Lemon SC, Roy J, Clark MA, Friedmann PD, Rakowski W. Classification and regression tree analysis in public health: methodological review and comparison with logistic regression. Soc Behav Med. 2003;26(3):172-81.

\section{Submit your next manuscript to BioMed Central and take full advantage of:}

- Convenient online submission

- Thorough peer review

- No space constraints or color figure charges

- Immediate publication on acceptance

- Inclusion in PubMed, CAS, Scopus and Google Scholar

- Research which is freely available for redistribution 